\title{
25 Research Suare \\ BUB1 Predicts Poor Prognosis and Immune Status in Liver Hepatocellular Carcinoma
}

wenbo qi

The Second Clinical Medical College of Lanzhou University

Yuping Bai

The Second Clinical Medical College of Lanzhou University

Le Liu

The Second Clinical Medical College of Lanzhou University

Hao Chen ( $\nabla$ ery_chenh@lzu.edu.cn )

Lanzhou University Second Hospital

\section{Research Article}

Keywords: LIHC, database, BUB1, prognosis, immunity.

Posted Date: October 13th, 2021

DOI: https://doi.org/10.21203/rs.3.rs-936503/v1

License: (c) (1) This work is licensed under a Creative Commons Attribution 4.0 International License. Read Full License

Version of Record: A version of this preprint was published at APMIS on March 7th, 2022. See the published version at https://doi.org/10.1111/apm.13219. 


\section{Abstract}

Background: Accurate assessment of the tumour immune microenvironment helps develop individualised immunotherapy regimens and screen dominant populations suitable for immunotherapy. Therefore, potential molecular markers were investigated to make an overall assessment of the immune microenvironment status of liver hepatocellular carcinoma (LIHC).

Methods: Differentially expressed genes (DEGs) in LIHC were extracted from the International Cancer Genome Consortium and The Cancer Genome Atlas databases. Gene set enrichment analysis was employed to assess the function of DEGs. Hub genes were identified using the STRING tool. The prognostic value of the hub genes was evaluated through Kaplan-Meier analysis and Cox regression. The correlation between genes and immunity was analysed using the TIMER tool. Further, tissue samples from 42 LIHC patients were collected for immunohistochemistry. HuH7 and SKP1 cells were analysed via western blotting, Cell Counting kit-8 assay, and Transwell assay.

Results: A total of 121 DEGs were identified, and DEGs were enriched in the epithelial-mesenchymal transition, hypoxia, myogenesis, and p53 pathways. A total of 20 hub genes were selected and a strong correlation was identified between these hub genes and prognosis. The expression of budding uninhibited by benzimidazoles 1 (BUB1), which is known to play a role in cancer progression, was found to be upregulated in LIHC (compared to normal tissues). Furthermore, BUB1 expression was strongly related to immune cells and immune checkpoint molecule expression. Immunohistochemistry indicated that BUB1 expression was higher in LIHC tissues than in normal liver tissues. Western blotting showed that $B U B 1$ expression was the highest in $\mathrm{HuH7}$ and SKP1 cells. BUB1 knockdown resulted in reduced proliferation and vertical migration ability of LIHC cells, and reduced the expression of phospho-SMAD family member 2 and phospho-SMAD family member 3 proteins. Immunohistochemistry showed that $B U B 1$ expression was accompanied by immune cell infiltration into LIHC tissues.

Conclusions: These results suggest that $B U B 1$ may serve as a potential prognostic biomarker for $\mathrm{LIHC}$ and as an indicator of its immune status.

\section{Introduction}

Liver hepatocellular carcinoma $(\mathrm{LIHC})$ is the seventh most common malignancy and ranks second in terms of global cancer-associated mortality (Sung et al., 2021). Thus, it poses a significant threat to human health on a global scale (Siegel et al., 2018). Approximately 780,000 individuals are diagnosed with LIHC every year, and a substantial proportion of these individuals are diagnosed with late-stage tumours (Torrecilla and Llovet, 2015). Despite significant progression in the treatment of LIHC recently, the survival probability of these patients is still below $20 \%$, and their prognosis is unsatisfactory (Lou et al., 2018). This prognosis status of patients is partly dependent on the surrounding microenvironment during tumour occurrence and development. The surrounding immune microenvironment can enable LIHC to escape immune recognition and attack by the body (Shlomai et al., 2014). The activation of 
inhibitory receptors and ligands, challenges associated with antigen presentation, and accumulation of immunosuppressive cell populations constitute the immunosuppressive microenvironment, thus promoting LIHC progression (Hato et al., 2014; Zhang et al., 2010). Therefore, research on genes related to tumour immune infiltration may aid the assessment of the immune microenvironment of LIHC, thereby facilitating patient stratification for immunotherapy and the development of personalised treatment approaches.

Budding uninhibited by benzimidazoles 1 ( $B U B 1$ ) is a kinase ensuring segregational fidelity in daughter cells during chromosomal segregation (Yu and Tang, 2005). It is encoded by the $B U B 1$ gene in humans. $B U B 1$ can be broadly segregated into three regions, i.e., a catalytic threonine or serine kinase domain, a scaffold for protein recruitment, and a tetratricopeptide repeat part (Kang et al., 2008). It is recognized as a tumour promoter in gastric cancer (Stahl et al., 2019). BUB1 was reported to be linked to forkhead box 03 in pancreatic cancer, which promotes pancreatic cancer development (Morris et al., 2015). The unfavourable prognostic effect of BUB1 expression in LIHC-identified by bioinformatic analysis-have been corroborated (Zhang et al., 2018). Though BUB1 activity has been explored, the mechanisms by which $B U B 1$ affects the prognosis and immune microenvironment in $\mathrm{LIHC}$ remain unclear. In this study, we first identified differentially expressed genes (DEGs) from the International Cancer Genome Consortium (ICGC) and The Cancer Genome Atlas (TCGA) databases and then identified the hub genes to further screen the immune-related gene BUB1. We observed that BUB1 was associated with prognostic and immune roles in $\mathrm{LIHC}$, thereby highlighting its relevance as a marker for LIHC immunotherapy.

\section{Materials And Methods}

\section{Datasets}

We downloaded datasets corresponding to 50 normal liver and $374 \mathrm{LIHC}$ tissue samples from The Cancer Genome Atlas Program (TCGA; https://cancergenome.nih.gov/). We also downloaded the Liver CancerFRANCE (LICA-FR) LIHC dataset from the International Cancer Genome Consortium database (ICGC; https://icgc.org); this dataset contains data pertaining to $150 \mathrm{LIHC}$ tissue samples and 11 paracancerous samples.

\section{Data pre-processing and DEGs identification}

The Affy package in R (Gautier et al., 2004) was used to analyse original data. After correcting for the inter-batch difference, original data were subjected to perform background correction, quality control and standardisation processing. The limma package in $\mathrm{R}$ (Ritchie et al., 2015) was applied to filter DEGs from the data set. The interception criteria for DEGs in the above database were $\mid \log$ fold change (FC) $\mid \geq 1$ and $p<0.05$ after adjustment. The results were visualised as a Venn diagram.

\section{Gene Ontology (GO) analysis, Kyoto Encyclopaedia of Genes and Genomes (KEGG) analysis, and Gene Set Enrichment Analysis (GSEA) of DEGs}


$\mathrm{GO}$ is one of the most frequently used tools for gene annotation, often employed for large-scale gene annotation. KEGG was used to identify pathways associated with DEGs. The distribution trends of genes and phenotypes were evaluated using GSEA. GO analysis, KEGG analysis, and GSEA were conducted using the clusterProfiler package in $\mathrm{R}$ (Yu et al., 2012).

\section{Construction of protein-protein interaction (PPI) network and hub gene identification}

The interaction between proteins encoded by the DEGs was evaluated by STRING tool (Szklarczyk et al., 2015). Then Cytoscape software (v3.7.1) (Smoot et al., 2011) was a platform for visualizing the PPI information. The top 20 genes were selected as hub genes using the cytoHubba plug-in (Chin et al., 2014).

\section{Prognostic validation of the hub genes}

Hub genes strongly correlated with prognosis were screened out by univariate and LASSO Cox regression analysis. The Survminer package (https://github.com/kassambara/survminer) and the survival package in R (https://github.com/therneau/surviva) were then used for mapping analysis. The association between genes and clinicopathological characteristics was analysed by logistic regression and receiver operating characteristic curve analyses. The selection criterion was $\mathrm{P}<0.05$.

\section{Expression andverification of hub genes}

The Gene Expression Profiling Interactive Analysis database (Tang et al., 2017) was used to analyse hub gene expression and related transforming growth factor $\beta$ (TGF $\beta$ ) signalling. The online tool TIMER (Li et al., 2017) was used to explore the correlation between hub genes and tumour-infiltrating immune cells.

\section{Tissue specimens}

A total of 42 paraffin-embedded and formalin-fixed LIHC tissue samples were collected from Lanzhou University Second Hospital. All patients had undergone curative resection surgeries. The study protocol was approved by the Institutional Ethics Committee of Lanzhou University Second Hospital. Written informed consent was obtained from all patients. Disease-free survival (DFS) was the period between the day of surgery and the date of recurrence.

\section{Immunohistochemistry (IHC)}

Tissues were serially sectioned into $4 \mu \mathrm{m}$-thick sections, heated in an oven at $67^{\circ} \mathrm{C}$ for $20 \mathrm{~min}$, and then dewaxed in alcohol and xylene. After antigen retrieval, the samples were treated with $3 \% \mathrm{H}_{2} \mathrm{O}_{2}$. Subsequently, the primary antibodies (BUB1, 1:50, Abcam, United States; CD3, CD4, CD8, CD20, CD56, CD68, CD163, ready-to-use, Maixin, Fujian) along with goat globulin ( $3 \mathrm{mg} / \mathrm{mL}$, Sigma, Aldrich) were used to incubate the sections at $4{ }^{\circ} \mathrm{C}$ overnight. Sections were then probed with anti-mouse/rabbit antibodies (1:100, Solarbio, Beijing) at $37^{\circ} \mathrm{C}$ for 30 min. Finally, 3, 3'-diaminobenzidine (Solarbio, Beijing) was used to stain the sections. 
Wherein a score of 0 indicated $<5 \%$ positive cells; a score of 1 indicated $6-20 \%$ positive cells; a score of 2 indicated $21-50 \%$ positive cells; and a score of 3 indicated $>50 \%$ positive cells.

\section{Western blotting (WB)}

Radioimmunoprecipitation assay buffer supplemented with phenyl methane sulfonyl fluoride was used to lyse tumour cells. After the lysate was centrifuged, the supernatant was collected for further analysis. After the protein concentration was measured, equal amounts of protein were loaded onto $10 \%$ SDSPAGE gels and transferred onto polyvinylidene fluoride membranes. Then, the membranes were blocked using $5 \%$ skimmed milk at $37^{\circ} \mathrm{C}$ for $2 \mathrm{~h}$ and were incubated with the primary antibodies (antiSMAD2/SMAD3, anti-BUB1, anti-PSMAD2/PSMAD3, 1:1,000, Abcam; anti-PDL1, 1:1,000, eBioscience, California; anti-GAPDH, 1:1,000, Proteintech, Chicago) at $4{ }^{\circ} \mathrm{C}$ overnight. Then, the anti-mouse/rabbit antibodies (1:3,000, Beyotime, Shanghai) were used to incubate the membranes at $37^{\circ} \mathrm{C}$ for $1 \mathrm{~h}$. The protein bands were detected by the chemiluminescence kit (Thermo Fisher, Shanghai).

\section{Transfection}

To silence the expression of endogenous $B U B 1$ in human $\mathrm{LIHC}$ cell lines, $2.5 \mu \mathrm{g}$ short hairpin RNA (shRNA) plasmids (Genechem, Shanghai) and $5 \mu \mathrm{L}$ Lipofectamine 6000 (Beyotime) were mixed in $500 \mu \mathrm{L}$ DMEM (Gibco, United States) medium for $5 \mathrm{~min}$. After incubation for $5 \mathrm{~min}$, the mixture was added to cells that had reached a confluence of 60 to $70 \%$ of 6 -well plate. The cells were used for further experiment after transfection for $24 \mathrm{~h}$. shRNA sequences were as follows: shBUB1, forward 5 '-CCGG CCTGGGTCAGAGTATAGATATCTCGAGATATCTATACTCTGACCCAGGTTTTTTG-3'; reverse, 5'AATTCAAAAACCTGGGTCAGAGTATAGATATCTCGAGATATCTATACTCTGACCCAGG-3'.

\section{Cell Counting Kit-8 (CCK-8) assay}

For the cell counting assay, 1,000 cells (HuH7 and SKP1 cells)/well were cultured in five replicate wells of a 96-well plate. The CCK-8 solution was sequentially added to every well and incubated for $2 \mathrm{~h}$ before detected. Absorbance 450 was detected by an instrument (TECAN, Infinite M200 Pro, Shanghai) at 12 h, 24 $\mathrm{h}, 48 \mathrm{~h}$, and $72 \mathrm{~h}$.

\section{Migration assays}

For migration assays, $1 \times 10^{5} \mathrm{HuH7}$ and SKP1 cells were independently resuspended in the medium (1\% foetal bovine serum) and seeded in the upper chamber of a Transwell chamber (Corning, Shanghai) placed in 24-well culture plates. Five hundred microlitres of medium ( $10 \%$ foetal bovine serum) was added to the bottom chamber. After incubation at $37^{\circ} \mathrm{C}$ for $24 \mathrm{~h}$, a cotton swab was used to remove the non-migrating cells on the membrane located at interface of the upper and lower chambers, and the migrated cells were fixed, stained, counted, and imaged. Six areas from each membrane were imaged from three independent wells.

\section{Statistical analysis}


The clusterProfiler package in R (Yu et al., 2012) and GraphPad Prism 8 (GraphPad Software Inc., Santiago) were used for data analysis. Student's $t$-tests were used to compare data between groups. Results are expressed as the mean \pm standard deviation. Significance was defined as $p<0.05$.

\section{Statement in the methods}

All methods were performed in accordance with the relevant guidelines and regulations.

\section{Results}

Identification and functional enrichment analysis of DEGs in LIHC

A total of 2,207 and 926 DEGs were screened from TCGA and ICGC, respectively. Of these, 121 DEGs overlapped, and the results are presented as a Venn diagram (Fig. 1).

DEGs were associated with chromosome segregation, nuclear division, and organelle fission by GO analysis (Fig. 2A). DEGs were associated with progesterone-mediated oocyte maturation, cell cycle, and oocyte meiosis by KEGG analysis (Fig. 2B). GSEA revealed that the DEGs were associated with the epithelial-mesenchymal transition (EMT), hypoxia (Fig. 2C), myogenesis, and p53 pathways (Fig. 2D). Collagen type I alpha 1 (COL1A1), COL3A1, COL5A1, COL6A3, v-Jun avian sarcoma virus 17 oncogene homolog $(J U M)$, and insulin-like growth factor-binding protein-1 (IGFBP1) play prominent roles in EMT and hypoxia (Fig. 2C). Cathepsin D (CTSD), JUN, tumour protein p53 (TP53), COL1A1, COL3A1, and COL6A3 play prominent roles in myogenesis as well as the p53 signalling pathway (Fig. 2D).

Selection and survival analysis of hub genes

The PPI network showed interactions between proteins encoded by DEGs (Fig. 3A). Twenty hub genes were identified (Fig. 3B), including ASPM, BIRC5, BUB1, BUB1B, CCNA2, CCNB1, CDK1, CENPF, DLGAP5, KIF20A, KIF23, MELK, NCAPG, NCAPH, NUSAP1, OIP5, PRC1, TOP2A, TPX2, and ZWINT (Table 1). 
Table 1

The 20 hub genes and their full names.

\begin{tabular}{|ll|}
\hline Gene & Full name \\
\hline ASPM & Abnormal spindle microtubule assembly \\
\hline BIRC5 & Baculoviral IAP repeat containing 5 \\
\hline BUB1 & BUB1 mitotic checkpoint serine/threonine kinase \\
\hline BUB1B & BUB1 mitotic checkpoint serine/threonine kinase B \\
\hline CCNA2 & Cyclin A2 \\
\hline CCNB1 & Cyclin B1 \\
\hline CDK1 & Cyclin-dependent kinase 1 \\
\hline CENPF & Centromere protein F \\
\hline DLGAP5 & DLG associated protein 5 \\
\hline KIF20A & Kinesin family member 20A \\
\hline KIF23 & Kinesin family member 23 \\
\hline MELK & Maternal embryonic leucine zipper kinase \\
\hline NCAPG & Non-SMC condensin I complex subunit G \\
\hline NCAPH & Non-SMC condensin I complex subunit H \\
\hline NUSAP1 & Nucleolar and spindle associated protein 1 \\
\hline OIP5 & Opa interacting protein 5 \\
\hline PRC1 & Protein regulator of cytokinesis 1 \\
\hline TOP2A & DNA topoisomerase II alpha \\
\hline TPX2 & TPX2 microtubule nucleation factor \\
\hline ZWINT & ZW10 interacting kinetochore protein \\
\hline
\end{tabular}

The correlation between these 20 hub genes and the poor prognosis of LIHC patients was analysed via Kaplan-Meier analysis ( $<$ 0.05) (Fig. 4). The 20 hub genes were then subjected to LASSO-Cox regression analysis to calculate the correlation coefficients. After cross-validation (Fig. 5A), hub genes strongly related to survival were finally identified that contained $B U B 1, C E N P F$, and PRC1 (Fig. 5B).

Expression of $B U B 1, C E N P F$, and PRC1 in LIHC 
The median expression of $B U B 1, C E N P F$ and $P R C 1$ in tumour and normal liver samples is shown in Figure 6. The expression of BUB1 (Fig. 6A), CENPF (Fig. 6B), and PRC1 (Fig. 6C) was found to be upregulated in LIHC as well as in other cancer types when compared to that in control samples. BUB1 (Fig. 6A), CENPF (Fig. 6B), and PRC1 (Fig. 6C) were upregulated in stage I-III of LIHC and downregulated in stage IV (Fig. 6). Multivariate and univariate Cox analyses suggested that $B U B 1$ expression was an independent prognostic factor of the overall survival of $\mathrm{LIHC}$ patients (Table $2, p<0.05$ ).

Table 2

Univariate and multivariate Cox regression analysis to identify a correlation between clinicopathological characteristics and BUB1 expression.

\begin{tabular}{|lllll|}
\hline Variables & Univariate analyses & \multicolumn{3}{l|}{ Multivariate analyses } \\
\cline { 2 - 5 } & HR $(95 \% \mathrm{Cl})$ & p-value & HR $(95 \% \mathrm{Cl})$ & p-value \\
\hline Age & $1.010(0.997-1.024)$ & 0.139 & $1.013(0.998-1.029)$ & 0.097 \\
\hline Sex & $0.816(0.573-1.163)$ & 0.260 & $1.001(0.671-1.493)$ & 0.997 \\
\hline Stage & $2.676(1.754-4.083)$ & $<0.001^{*}$ & $2.267(1.458-3.526)$ & $<0.001^{*}$ \\
\hline Metastasis & $2.479(1.695-3.897)$ & 0.005 & $4.347(2.738-6.535)$ & 0.002 \\
\hline BUB1 & $1.429(1.225-1.668)$ & $<0.001^{*}$ & $1.389(1.166-1.655)$ & $<0.001^{*}$ \\
\hline * Significant findings. & & & \\
\hline
\end{tabular}

Correlation of $B U B 1$ with immunity and TGF $\beta$ signalling

The expression of $B U B 1$ (Fig. 7A), CENPF (Fig. 7B), and PRC1 (Fig. 7C) was correlated with the counts of CD4+/CD8+ T cells, B cells, dendritic cells, macrophages, and neutrophils $(p<0.05)$ (Fig. 7). Furthermore, $B U B 1$ expression was associated with all immune checkpoints in $\mathrm{LIHC}$, and was strongly associated with CD274 expression (programmed cell death 1 ligand 1, PD-L1) $(p<0.05)$ (Fig. 7D).

Previous reports have provided evidence regarding the role of $B U B 1$ in mediating TGF $\beta$-dependent signalling, in addition to its established function in chromosome cohesion and cell cycle regulation (Nyati et al., 2015; Nyati et al., 2020). In LIHC, BUB1 expression was correlated with TGF- $\beta 1$, TGF $\beta 2$, TGF $\beta 3$, TGF $\beta$ receptor (TGFBR) 1, SMAD2, SMAD3, and SMAD4 ( $<0.05)$ (Fig. 8).

\section{Validation of the role of BUB1 in LIHC}

$\mathrm{IHC}$ indicated that BUB1 was upregulated in $\mathrm{LIHC}$ tissues (compared to paracancerous tissues) (Fig. 9A). $B U B 1$ expression was associated with poor DFS ( $<$ < 0.05) (Fig. 9B). BUB1 levels were higher in HuH7 and SKP1 cells than in other LIHC cell lines (Fig. 9C). 
Functional enrichment analysis suggested that BUB1 may promote LIHC progression. MTT and Transwell migration assays were performed in $\mathrm{HuH7}$ and SKP1 cells to validate the functional significance of $B U B 1$. Our results revealed that $B U B 1$ knockdown significantly reduced the proliferation (Fig. 10A) and vertical migration ability of LIHC cells ( $<<0.05)$ (Fig. 10B).

To evaluate the role of $B U B 1$ in TGF $\beta$ signalling, the levels of TGF $\beta$ signalling-related proteins were evaluated via WB. BUB1 knockdown downregulated the expression of phospho-SMAD (p-SMAD) 2 and pSMAD3 expression ( $<$ 0.05) (Fig. 10C). BUB1 knockdown did not result in a decrease in PD-L1 expression. IHC for BUB1 and immune cell markers (CD3, CD4, CD8, CD20, CD56, CD68, and CD163) in the serial section of LIHC tissues is shown in Figure 11A. BUB1 expression was correlated with CD4, CD8, CD56 (NCAM1), CD68, and CD163 ( $p$ 0.05) (Fig. 11B) in LIHC. These results verified the association of $B U B 1$ expression with immune cell infiltration in LIHC.

\section{Discussion}

There is clear evidence that the immunosuppressive microenvironment of LIHC facilitates immune escape and tolerance through a variety of mechanisms; this feature makes LIHC a challenging candidate for immunotherapy (Fu et al., 2019). Emerging evidence suggests that counteracting these immunosuppressive mechanisms may significantly alter the clinical outcomes of LIHC, and will aid in conducting in-depth investigations on the correlation between the occurrence and prognosis of LIHC and immune genes (Khemlina et al., 2017). Currently-aided by bioinformatic analysis-cancer immunotherapy (Floudas et al., 2019) is ushering in a new epoch of anti-cancer therapies. In the present study, the gene expression profiles in LIHC and associated pathological mechanisms were investigated using bioinformatic tools. We identified BUB1 as being closely related to tumour cell proliferation, metastasis, and immunity in LIHC. We found that research on its mechanism of action in LIHC is currently scarce; therefore, we performed a comprehensive analysis of its expression, clinical relevance, potential prognostic and immunologic value in LIHC.

$B U B 1$ is verified as a key protein in mitosis, and it has been recognised as an oncogene in gastric and breast cancer (Stahl et al., 2019; Han et al., 2015). In the current study, BUB1 expression was found to be upregulated in LIHC tissue samples. Further, BUB1 was significantly associated with the clinical stage of LIHC. Survival analyses indicated that BUB1 was associated with reduced overall survival. Further, $B U B 1$ knockdown resulted in reduced proliferation and vertical migration of LIHC cells. Therefore, we speculated that BUB1 may act as an oncogene, thereby promoting LIHC progression.

Common pathways that are deregulated in $\mathrm{LIHC}$ include the cell cycle, immune response, DNA replication, DNA repair, p53 and TGF $\beta$ signalling. TGF $\beta$ signalling is known to have a vital influence on the progression of LIHC (Lin et al., 2015) through the regulation of immune cell survival, development, proliferation, and differentiation (Li and Flavell, 2008; Chen and Ten Dijke, 2016). TGF $\beta$ signalling reduces CD4+/CD8+ T cell, natural killer cell, and macrophage counts. It also promotes the generation of regulatory T cells by upregulating FoxP3 via the SMAD2/SMAD3 pathway (Achyut and Yang, 2011). 
Recently, combinatorial therapy with anti-PD-L1 immune checkpoint inhibitors (ICls) and systemic TGF $\beta R 1$ inhibitors (e.g., galunisertib) or anti-TGF $\beta$ antibodies was shown to promote the infiltration of $T$ cells into the tumour core, thereby inducing tumour regression and anti-tumour immunity (Mariathasan et al., 2018). BUB1, as a kinase, is reported to have a vital impact on TGF $\beta$-dependent signalling (Nyati et al., 2015). $B U B 1$ is the engine of TGF $\beta$ signalling (Moustakas, 2015). Because of the immunosuppressive effect of TGF $\beta$-dependent signalling in LIHC and the role of $B U B 1$ in TGF $\beta$ signalling, we speculated that $B U B 1$ might be a key molecule in the TGF $\beta$-based immune regulation in LIHC. Our results verified the hypothesis that $B U B 1$ expression was related to immune cell counts and immune checkpoint molecule expression in $\mathrm{LIHC}$. A previous study also suggested $B U B 1$ as a new indicator of the $\mathrm{ICl}$ response in lung cancer (Pabla et al., 2019). Thus, we predicted that BUB1 might also be an important gene for ICls therapy and might provide insights that enable us to identify novel targets for immune therapies for LIHC. We confirmed that $B U B 1$ was associated with immune cell infiltration in $L I H C$, highlighting its influence on LIHC immune status. In a subsequent study, we will verify the involvement of $B U B 1$ in immune regulation in vivo.

In summary, BUB1 expression may predict poor prognosis and immune status of LIHC. The current study provides a theoretical basis for further exploring the specific regulatory mechanisms associated with BUB1 in LIHC.

\section{Declarations}

\section{Conflict of Interest}

The authors declare that the research was conducted in the absence of any commercial or financial relationships that could be construed as a potential conflict of interest.

\section{Funding}

This work was supported by Key Talents Project of Gansu Province (No. 2019RCXM020).

\section{Author Contributions}

Wenbo Qi and Yuping Bai conducted the experiments and wrote the manuscript. All authors contributed to the article and approved the submitted version.

\section{Acknowledgments}

We would like to express our gratitude to Editage (www.editage.cn) for English language editing. 


\section{Data Availability Statement}

All data generated or analysed during this study are included in this published article.

\section{References}

1. Achyut, B., and Yang, L. (2011). Transforming growth factor-beta in the gastrointestinal and hepatic tumor microenvironment. Gastroenterology 141, 1167-1178. doi: 10.1053/j.gastro.2011.07.048.

2. Chen, Q., Xia, J., Li, W., Shen, L., Huang, T., and Wu, P. (2018). Examining the key genes and pathways in hepatocellular carcinoma development from hepatitis B virus-positive cirrhosis. Mol. Med. Rep. 18, 4940-4950. doi: 10.3892/mmr.2018.9494.

3. Chen, W., and Dijke, P. (2016). Immunoregulation by members of the TGF $\beta$ superfamily. Nat. Rev. Immunol. 16, 723-740. doi: 10.1038/nri.2016.112.

4. Chin, C., Chen, S., Wu, H., Ho, C., Ko, M., and Lin, C. (2014). CytoHubba: identifying hub objects and sub-networks from complex interactome. BMC Syst. Biol. 8, S11-S16. doi: 10.1186/1752-0509-8-S4S11.

5. Floudas, C. S., Brar, G., and Greten, T. F. (2019). Immunotherapy: current status and future perspectives. Dig. Dis. Sci. 64, 1030-1040. doi: 10.1007/s10620-019-05516-7.

6. Fu, Y., Liu, S., Zeng, S., and Shen, H. (2019). From bench to bed: the tumor immune microenvironment and current immunotherapeutic strategies for hepatocellular carcinoma. J. Exp. Clin. Cancer Res. 38, 396-417. doi: 10.1186/s13046-019-1396-4.

7. Gautier, L., Cope, L., Bolstad, B., and Irizarry, R. (2004) Affy-analysis of Affymetrix GeneChip data at the probe level. Bioinformatics 20, 307-315. doi: 10.1093/bioinformatics/btg405.

8. Han, J., Han, Y., Park, G., Kim, S., and Lee, C. (2015). Bub1 is required for maintaining cancer stem cells in breast cancer cell lines. Sci. Rep. 5, 15993-16000. doi: 10.1038/srep15993.

9. Hato, T., Goya, L., Greten, T., Duda, D., and Zhu, A. (2014). Immune checkpoint blockade in hepatocellular carcinoma: current progress and future directions. Hepatology 60, 1776-1782. doi: 10.1002/hep.27246.

10. Khemlina, G., Ikeda, S., and Kurzrock, R. (2017). The biology of hepatocellular carcinoma: implications for genomic and immune therapies. Mol. Cancer 16, 149-158. doi: 10.1186/s12943017-0712-х.

11. Kang, J. S., Yang, M. J., Li, B., Qi, W., Zhang, C., and Shokat, K., et al. (2008). Structure and Substrate Recruitment of the Human Spindle Checkpoint Kinase Bub1. Mol. Cel/32, 394-405. doi: 10.1016/j.molcel.2008.09.017.

12. Li, M., and Flavell, R. (2008). TGF- $\beta$ : a master of all T cell trades. Cell 134, 392-404. doi: 10.1016/j.cell.2008.07.025.

13. Li, T., Fan, J., Wang, B., Traugh, N., Chen, Q., and Liu, J. S., et al. (2017). TIMER: a web server for comprehensive analysis of tumor-infiltrating immune cells. Cancer Res. 77, e108-118. doi: 
10.1158/0008-5472.CAN-17-0307.

14. Lin, T. H., Shao, Y. Y., Chan, S. Y., Huang, C. Y., Hsu, C. H., and Cheng, A. L., et al. (2015).

High serum transforming growth factor-beta1 Levels Predict Outcome in Hepatocellular Carcinoma Patients Treated with Sorafenib. Clin. Cancer Res. 21, 3678-3684. doi: 10.1158/1078-0432.CCR-141954.

15. Lou, W., Liu, J., Gao, Y., Zhong, G., Ding, B., and Xu, L., et al. (2018). MicroRNA regulation of liver cancer stem cells. Am. J. Cancer Res. 8, 1126-1141. doi: 10.1016/j.clinre.2015.06.016.

16. Mariathasan, S., Turley, S. J., Nickles, D., Castiglioni, A., Yuen, K., and Wang, Y., et al. (2018). TGF $\beta$ attenuates tumour response to PD-L1 blockade by contributing to exclusion of T cells. Nature 554, 544-548. doi: 10.1038/nature25501.

17. Morris, B., Willcox, D., Donlon, T., and Willcox, B. (2015). FOX03: A Major Gene for Human LongevityA Mini-Review. Gerontology 61, 515-525. doi: 10.1159/000375235.

18. Moustakas, A. (2015). The mitotic checkpoint protein kinase BUB1 is reported as an engine in the TGF- $\beta$ signaling apparatus. Sci. Signal. 8, 359. doi: 10.1126/scisignal.aaa4636.

19. Nyati, S., Gregg, B. S., Xu, J., Young, G., Kimmel, L., and Nyati, M. K., et al. (2020). TGFBR2 mediated phosphorylation of BUB1 at Ser-318 is required for transforming growth factor-b signaling. Neoplasia 22, 163-178. doi: 10.1016/j.neo.2020.02.001.

20. Nyati, S., Schinske-Sebolt, K., Pitchiaya, S., Chekhovskiy, K., Chator, A., and Chaudhry, N., et al. (2015). The kinase activity of the Ser/Thr kinase BUB1 promotes TGF- $\beta$ signaling. Sci. Signal. 8, 358. doi: 10.1126/scisignal.2005379.

21. Pabla, S., Conroy, J. M., Nesline, M. K., Glenn, S. T., Papanicolau-Sengos, A., and Burgher, B., et al. (2019). Proliferative potential and resistance to immune checkpoint blockade in lung cancer patients. J. Immunother. Cancer 7, 27-40. doi: 10.1186/s40425-019-0506-3.

22. Ritchie, M. E., Phipson, B., Wu, D. I., Hu, Y., Law, C. W., and Shi, W., et al. (2015). limma powers differential expression analyses for RNA-sequencing and microarray studies. Nucleic Acids Res. 43, e47-e59. doi: 10.1093/nar/gkv007.

23. Shlomai, A., Jong, Y., and Rice, C. (2014). Virus associated malignancies: the role of viral hepatitis in hepatocellular carcinoma. Semin. Cancer Biol. 26, 78-88. doi: 10.1016/j.semcancer.2014.01.004.

24. Siegel, R., Miller, K., and Jemal, A. (2018). Cancer statistics, 2018. CA Cancer J. Clin. 68, 7-30. doi: $10.3322 /$ caac. 21442 .

25. Smoot, E., Ono, K., Ruscheinski, J., Wang, P., and Ideker, T. (2011). Cytoscape 2.8: new features for data integration and network visualization. Bioinformatics 27, 431-432. doi: 10.1093/bioinformatics/btq675.

26. Stahl, D., Braun, M., Gentles, A., Lingohr, P., Walter, A., and Kristiansen, G., et al. (2017). Low BUB1 expression is an adverse prognostic marker in gastric adenocarcinoma. Oncotarget 8, 76329-76339. doi: 10.18632/oncotarget.19357.

27. Sung, H., Ferlay, J., Siegel, R. L., Laversanne, M., Soerjomataram, I., and Jemal, A., et al. (2021). Global Cancer Statistics 2020: GLOBOCAN Estimates of Incidence and Mortality Worldwide for 36 
Cancers in 185 Countries. CA Cancer J. Clin. 71, 209-249. doi: 10.3322/caac.21660.

28. Szklarczyk, D., Franceschini, A., Wyder, S., Forslund, K., Heller, D., and Huerta-Cepas, J., et al. (2015). STRING v10: protein-protein interaction networks, integrated over the tree of life. Nucleic Acids Res. 43, D447-D452. doi: 10.1093/nar/gku1003.

29. Tang, Z., Li, C., Kang, B., Gao, G., Li, C., and Zhang, Z. (2017). GEPIA: a web server for cancer and normal gene expression profiling and interactive analyses. Nucleic Acids Res. 45, W98-W102. doi: $10.1093 /$ nar/gkx247.

30. Torrecilla, S., and Llovet, J. (2015). New molecular therapies for hepatocellular carcinoma. Clin. Res. Hepatol. Gastroenterol. 39, 80-86. doi: 10.1016/j.clinre.2015.06.016.

31. Yu, H., and Tang, Z. (2005). Bub1 multitasking in mitosis. Cell Cycle 4, 262-265. doi: $10.4161 /$ cc.4.2.1487.

32. Yu, G., Wang, L., Han, Y., and He, Q. (2012). ClusterProfiler: an R package for comparing biological themes among gene clusters. OMICS 16, 284-287. doi: 10.1089/omi.2011.0118.

33. Zhang, H. H., Mei, M. H., Fei, R., Liu, F., Wang, J. H., and Liao, W. J., et al. (2010). Regulatory T cells in chronic hepatitis $B$ patients affect the immunopathogenesis of hepatocellular carcinoma by suppressing the antitumour immune responses. J. Viral. Hepat. 17, 34-43. doi: 10.1111/j.13652893.2010.01269.

34. Zhang, L., Huang, Y., Ling, J., Zhuo, W., Yu, Z., and Shao, M., et al. (2018). Screening and function analysis of hub genes and pathways in hepatocellular carcinoma via bioinformatics approaches. Cancer Biomark. 22, 511-521. doi: 10.3233/CBM-171160.

\section{Figures}

\section{Figure 1}


Identification of DEGs in the LIHC datasets. A total of 2,086 DEGs were screened from TCGA dataset, and 805 DEGs were screened from the ICGC dataset. The Venn diagram revealed a total of 121 DEGs overlapping between TCGA and ICGC data.

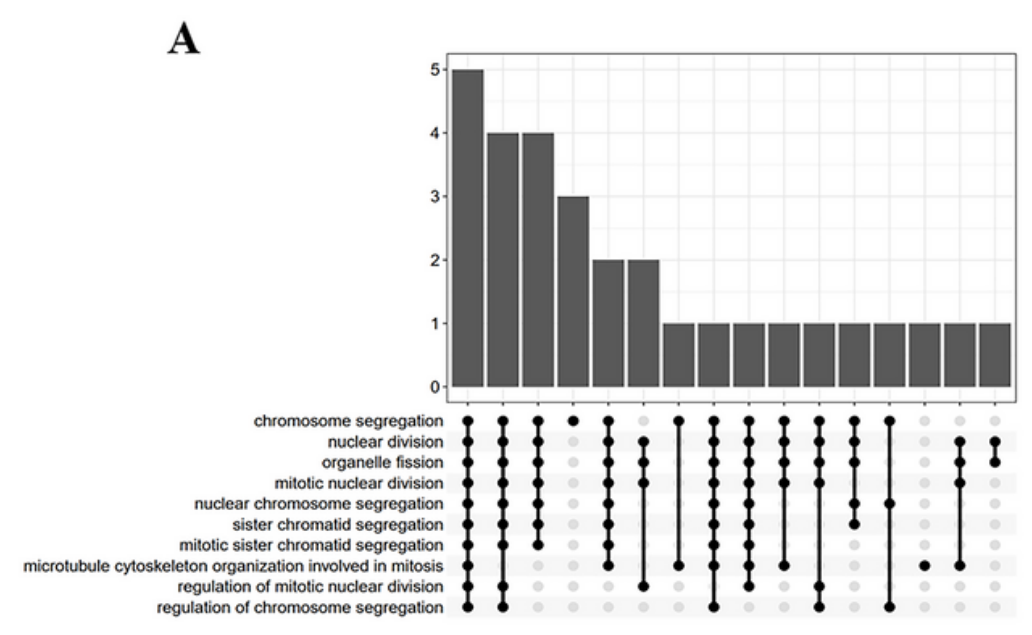

C

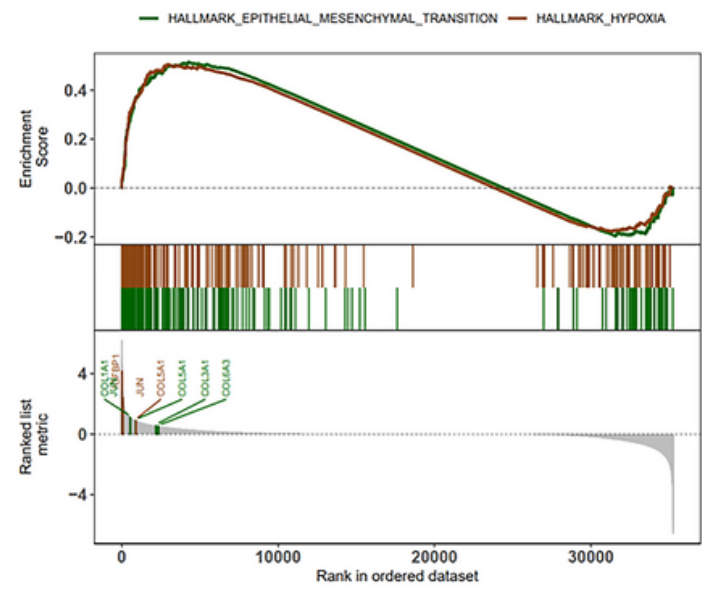

B

D

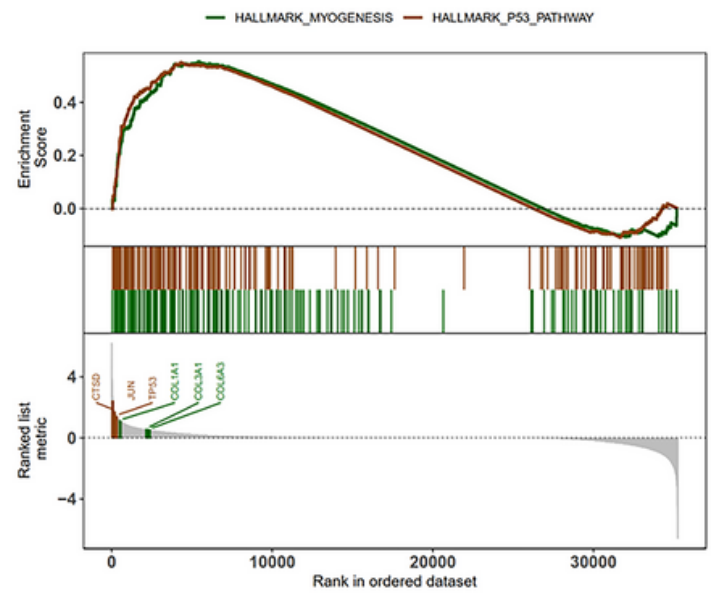

\section{Figure 2}

Functional enrichment analysis of DEGs. (A-B) Upset plots showed the number of genes that overlapped between different combinations of function pathways. GO analysis (A) indicated that DEGs were mainly enriched in terms related to chromosome segregation, nuclear division, organelle fission, mitotic nuclear division, nuclear chromosome segregation, and sister chromatid segregation. KEGG pathway analysis (B) indicated that DEGs were mainly enriched in terms associated with the cell cycle, progesterone-mediated oocyte maturation, and oocyte meiosis pathways. (C-D) GSEA revealed that the enriched pathways mainly included the EMT, hypoxia, myogenesis, and p53 pathways. COL1A1, COL3A1, COL5A1, COL6A3, COL6A3, JUN, and IGFBP1 play prominent roles in EMT and hypoxia. CTSD, JUN, TP53, COL1A1, COL3A1, and COL6A3 play prominent roles in myogenesis and p53 signalling pathways. 
A

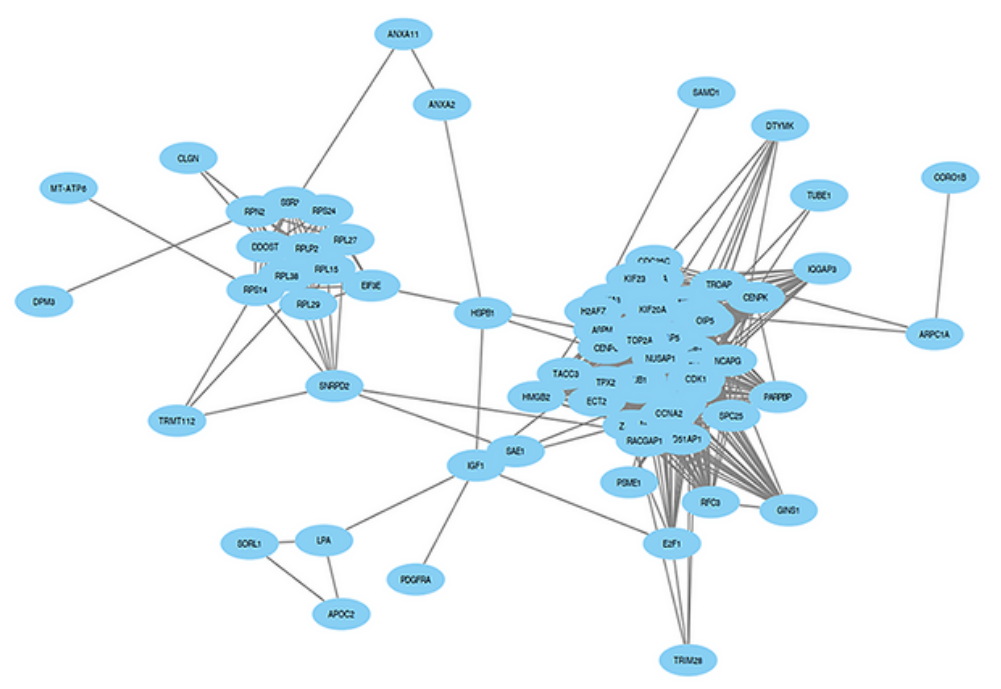

B

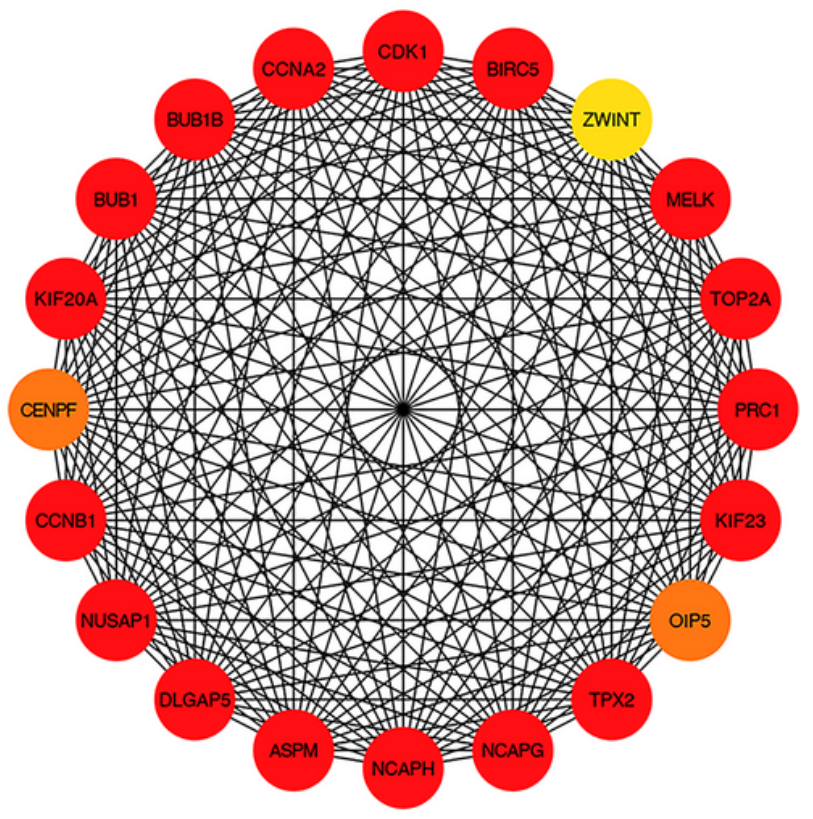

Figure 3

PPI analysis of DEG-encoded proteins and mapping of the hub gene module. (A) The PPI network showed the interaction between the proteins encoded by DEGs. (B) The 20 hub genes associated with LIHC are shown. The hub genes included ASPM, BIRC5, BUB1, BUB1B, CCNA2, CCNB1, CDK1, CENPF, DLGAP5, KIF20A, KIF23, MELK, NCAPG, NCAPH, NUSAP1, OIP5, PRC1, TOP2A, TPX2, and ZWINT. 



$\mathrm{PRC1}->9.8(145)-<9.8(223)$

ASPM $->9.65(162)-<9.65(206)$
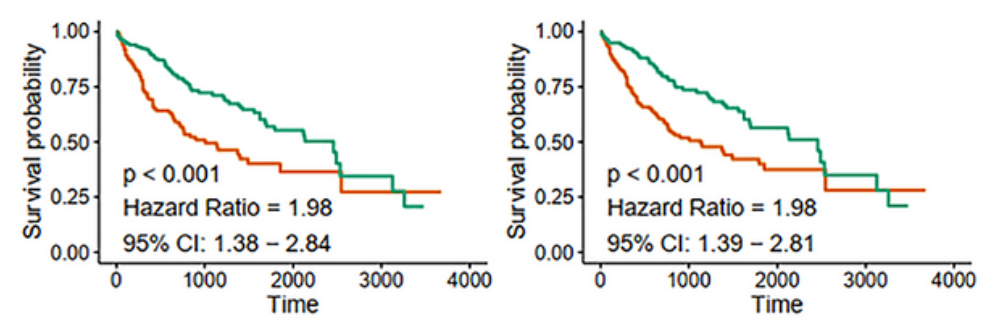

NCAPG $->9.25(116)-<9.25(252)$

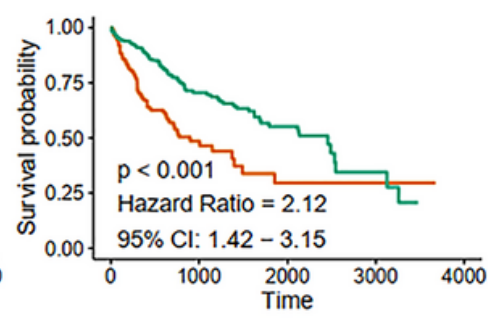

CENPF $->10.59(111)-<10.59(257)$

TPX2 $->10.62(134)-<10.62(234)$

CDK1 $->9.21(168)-<9.21(200)$
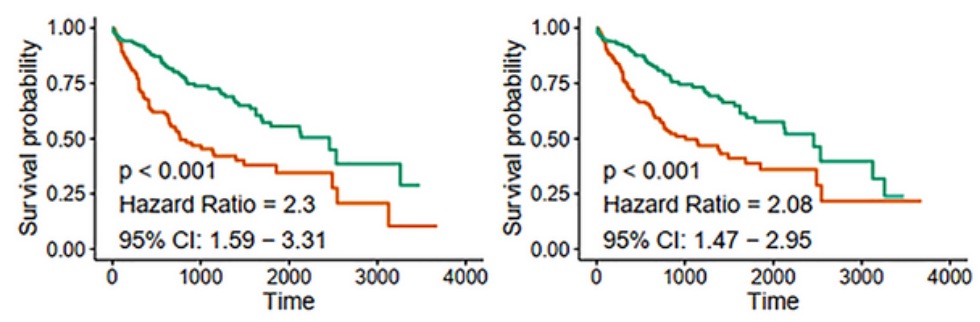

$\mathrm{NCAPH}->7.84(169)-<7.84(199)$
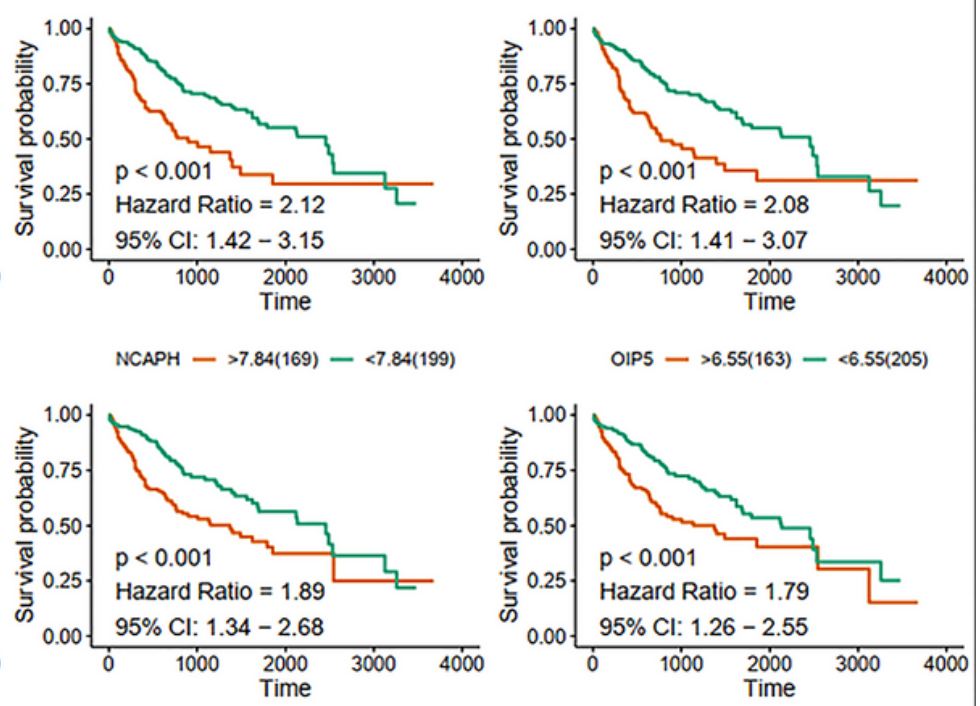

NUSAP1 $->9.51(198)-<9.51(170)$

CCNA2 $->8.1(218)-<8.1(150)$

BUB1B $->8.48(122)-<8.48(246)$

ZWINT $->9.82(134)-<9.82(234)$
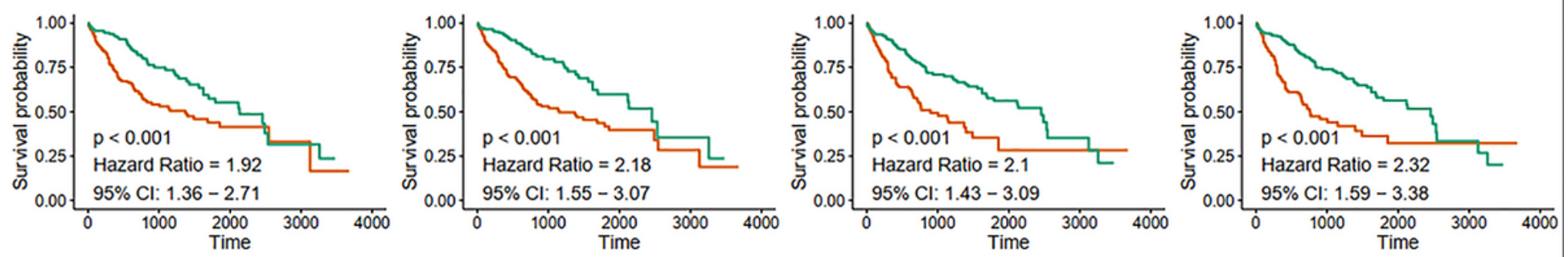

\section{Figure 4}

Kaplan-Meier survival curves for the TCGA cohort based on the hub genes. LIHC patients with high expression of hub genes had a poorer prognosis than those with the low expression of these genes (all $p$ $<0.05)$. 
A

$\begin{array}{llllllllllllllllllll}19 & 19 & 18 & 17 & 17 & 16 & 15 & 11 & 8 & 8 & 6 & 4 & 4 & 4 & 3 & 3 & 3 & 3 & 2 & 2\end{array}$

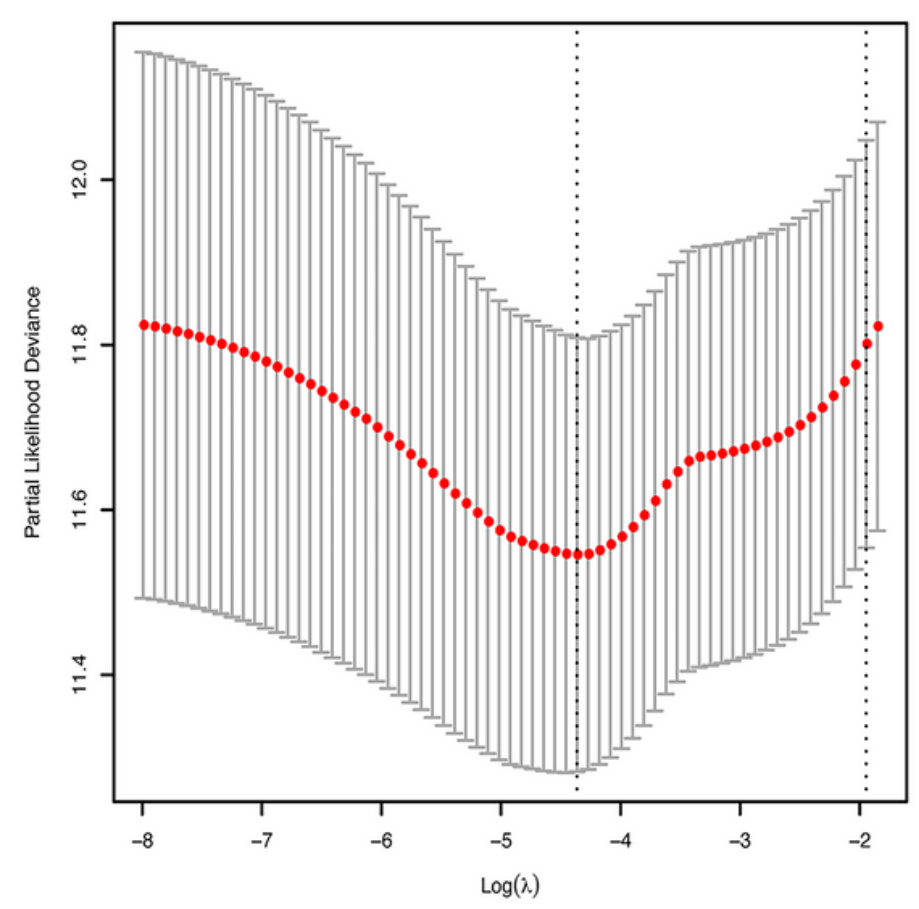

B

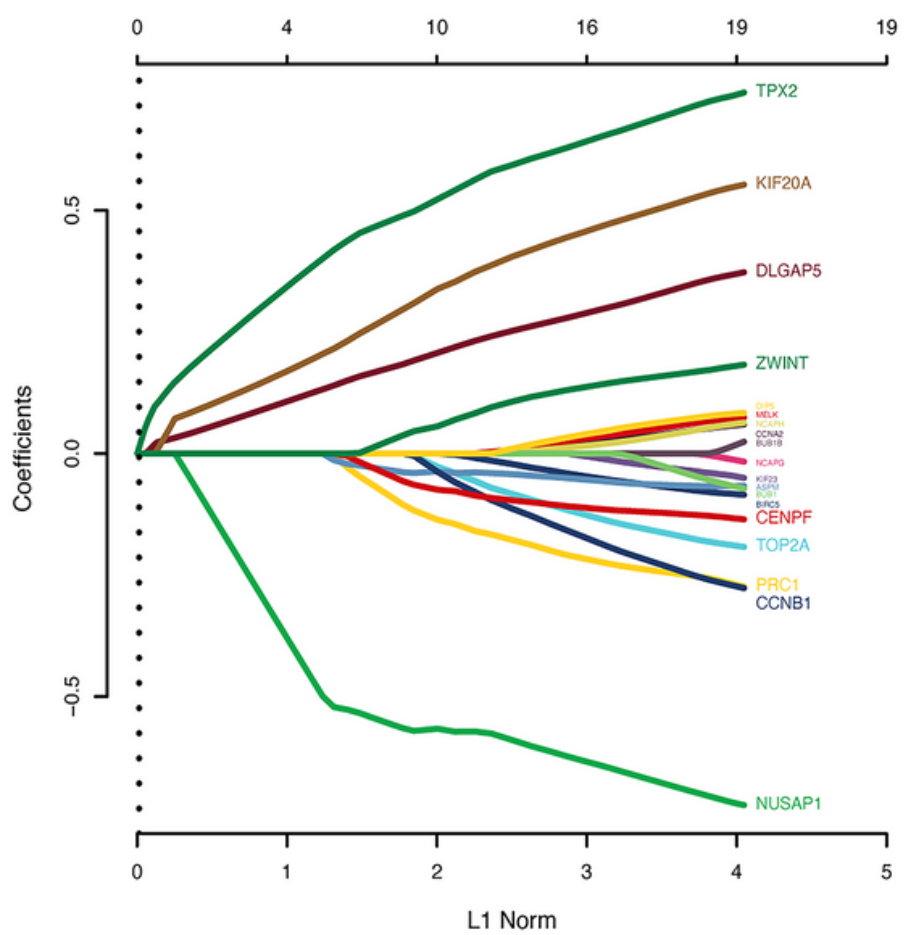

Figure 5

Lasso Cox survival analysis of the 20 hub genes associated with LIHC. (A) Cross-validation was used to select the tuning parameter $\lambda$ value. (B) Regression coefficient map of the 20 hub genes in the LASSO model was constructed based on the optimal $\lambda$. The hub genes strongly related to survival were identified; these included BUB1, CENPF, and PRC1. 
A

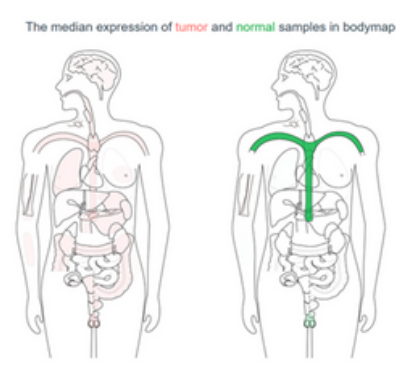

B

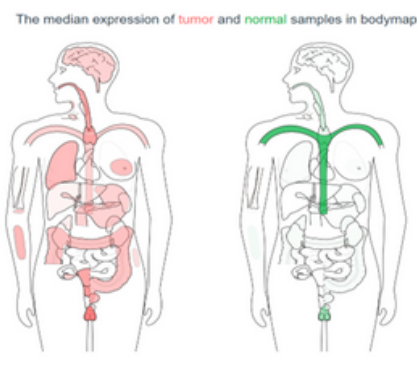

C

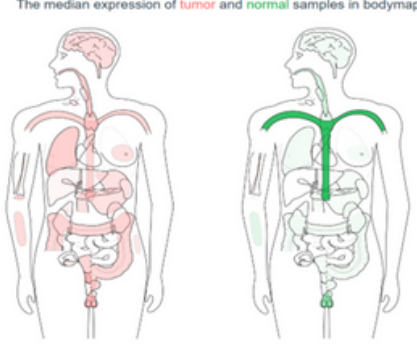

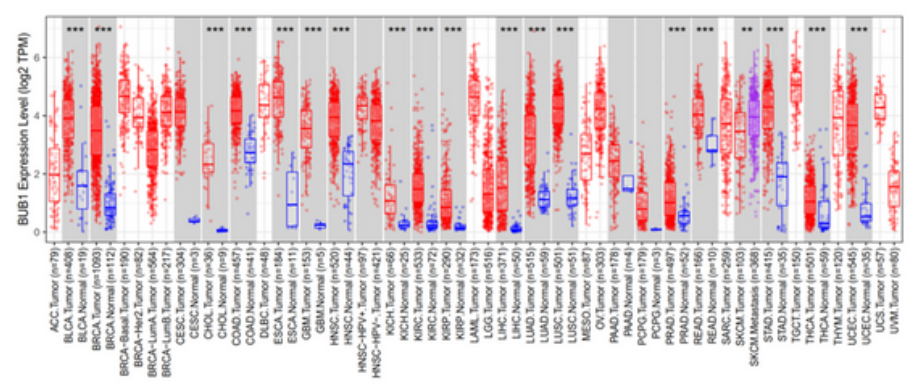
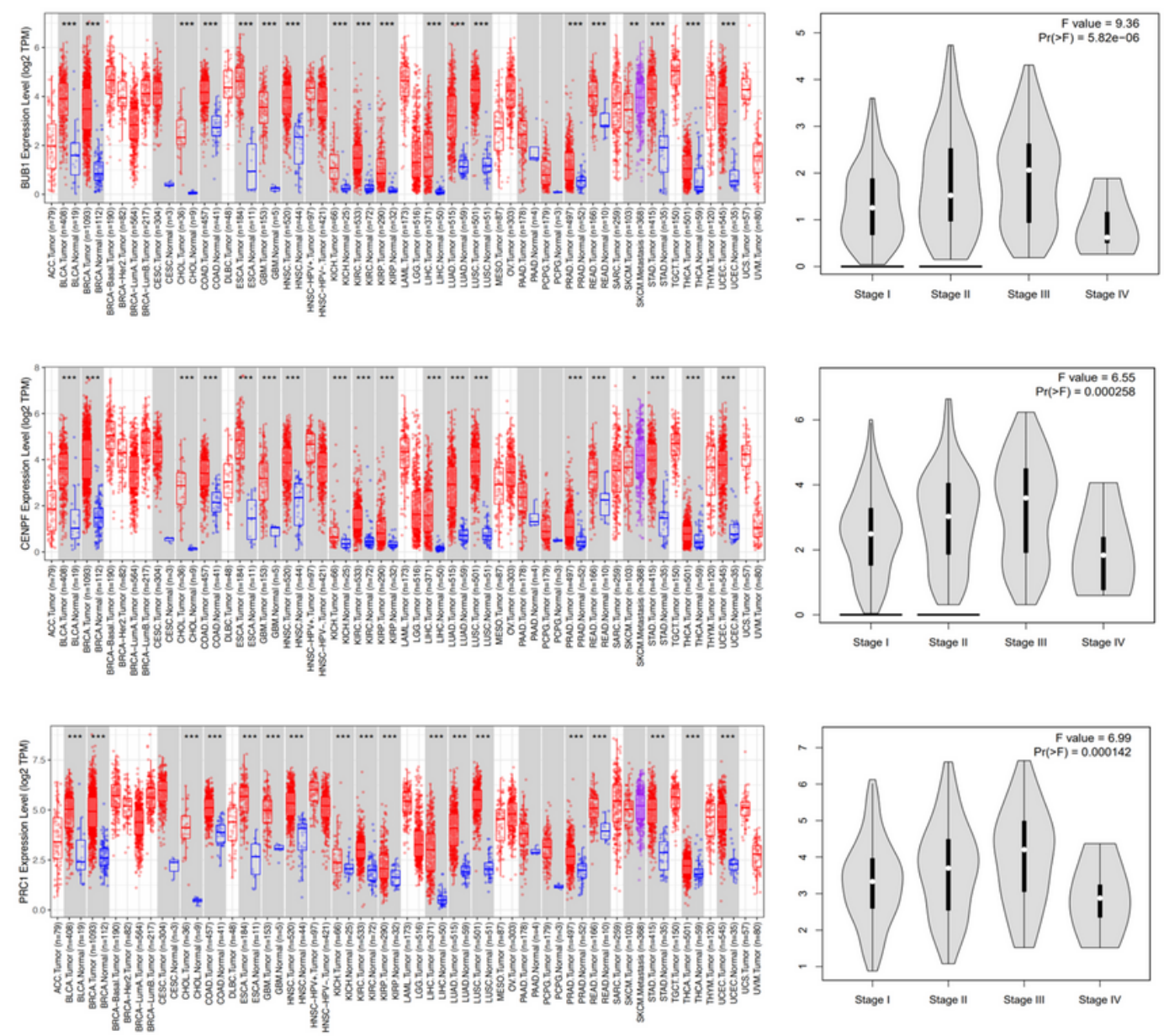

Figure 6

The expression of BUB1 (A), CENPF (B), and PRC1 (C) in LIHC. The expression of BUB1, CENPF and PRC1 was upregulated in LIHC compared to that in the control group. The expression of BUB1, CENPF, and PRC1 increased in stage I-III of LIHC and decreased in stage IV. 
A

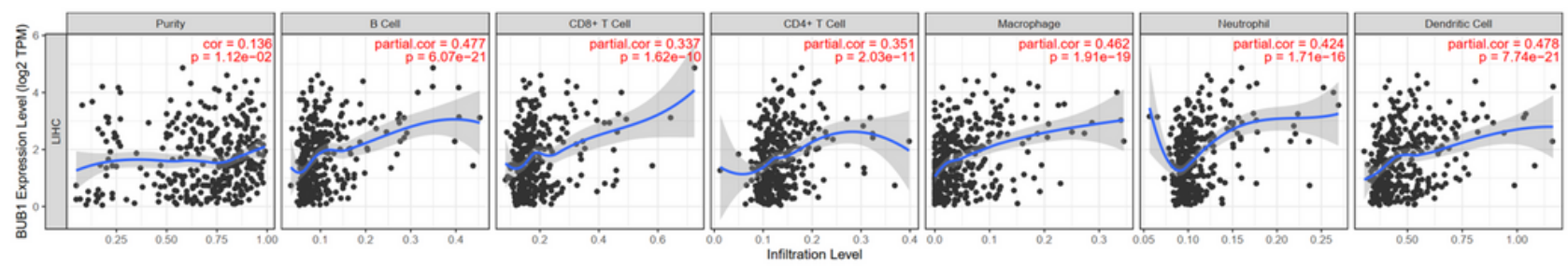

B

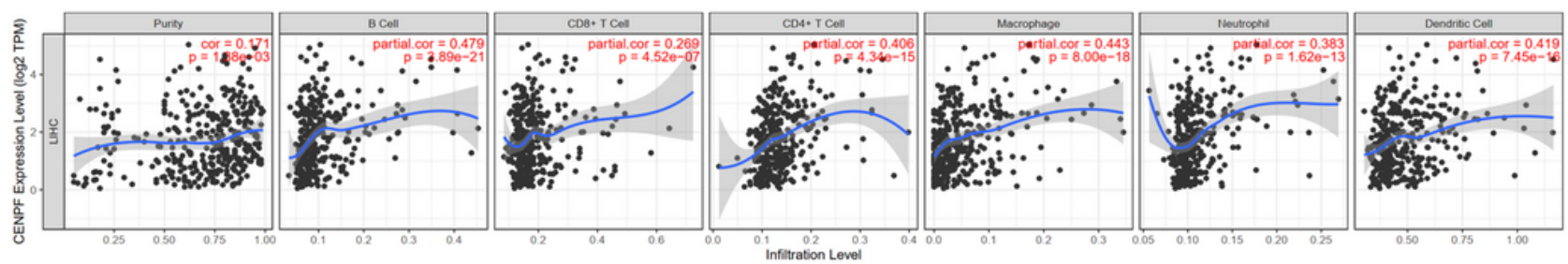

C

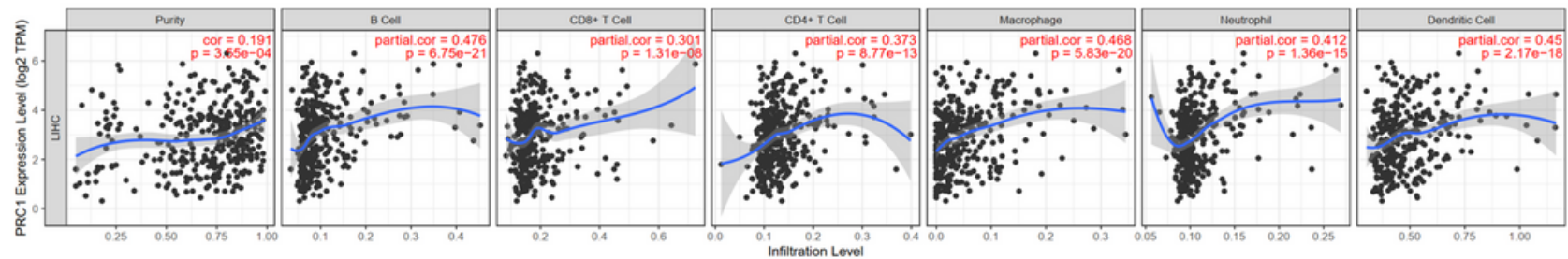

D

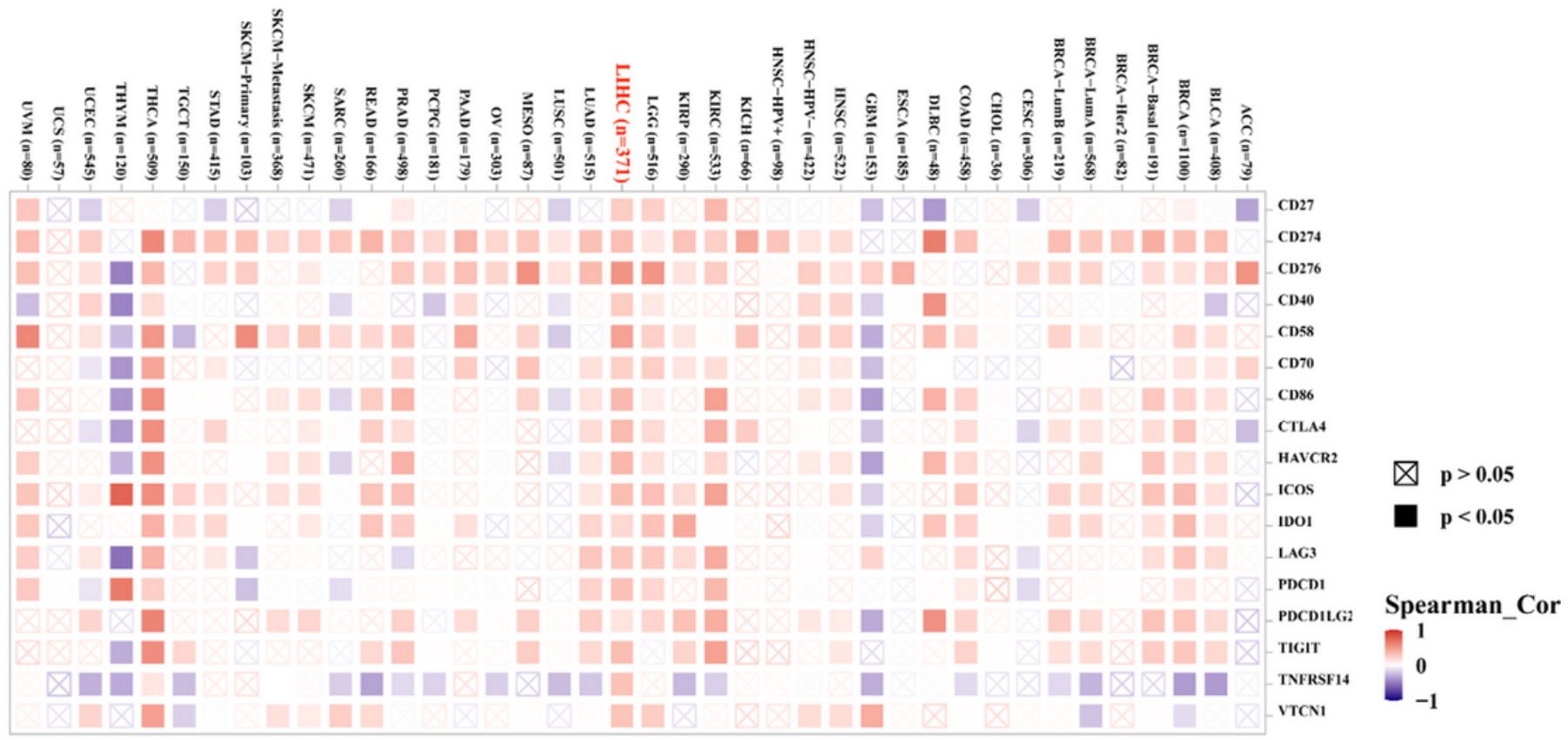

\section{Figure 7}

Correlation between BUB1, CENPF, and PRC1 expression and immune infiltration in LIHC tissues. BUB1 $(A)$, CENPF (B), and PRC1 (C) expression were correlated with tumour purity as well as with the counts of CD4+/CD8+ T cells, B cells, macrophages, neutrophils, and dendritic cells ( $p<0.05)$. (D) BUB1 expression was also associated with that of all immune checkpoint molecules in LIHC $(p<0.05)$. 

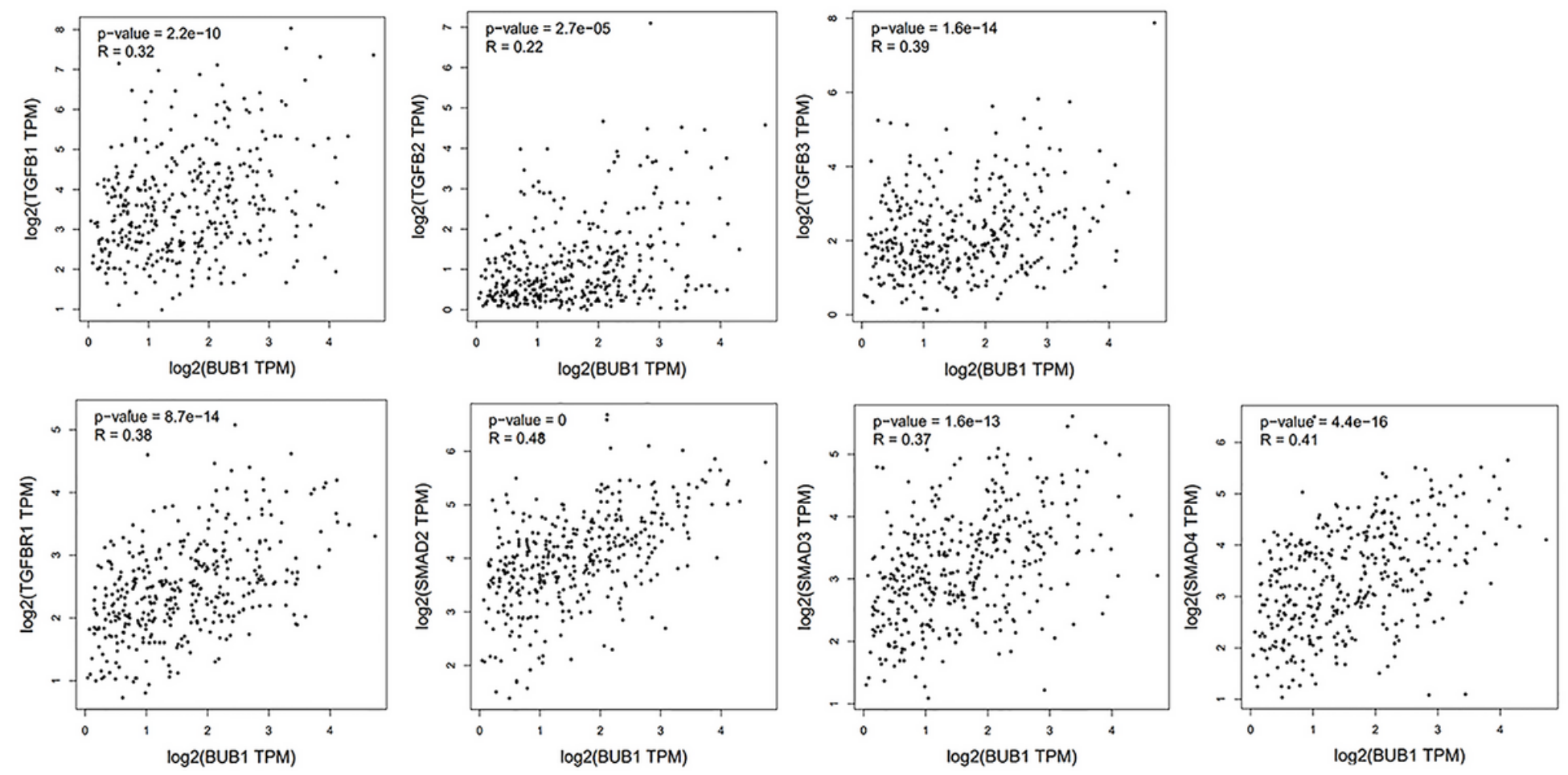

Figure 8

Correlation between BUB1 and TGF $\beta$ signalling in LIHC. BUB1 expression was correlated with that of TGF 1 1, TGFß2, TGFß3, TGFßR1, SMAD2, SMAD3, and SMAD4 $(p<0.05)$.

A

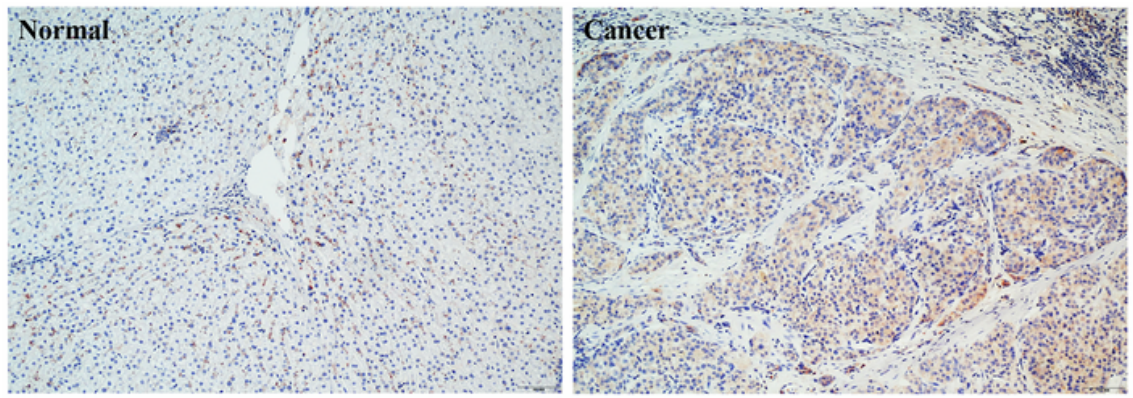

B

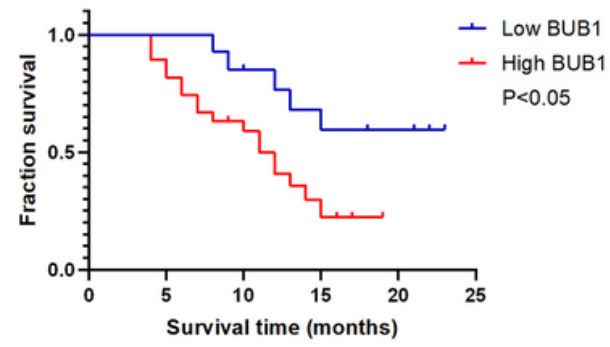

B

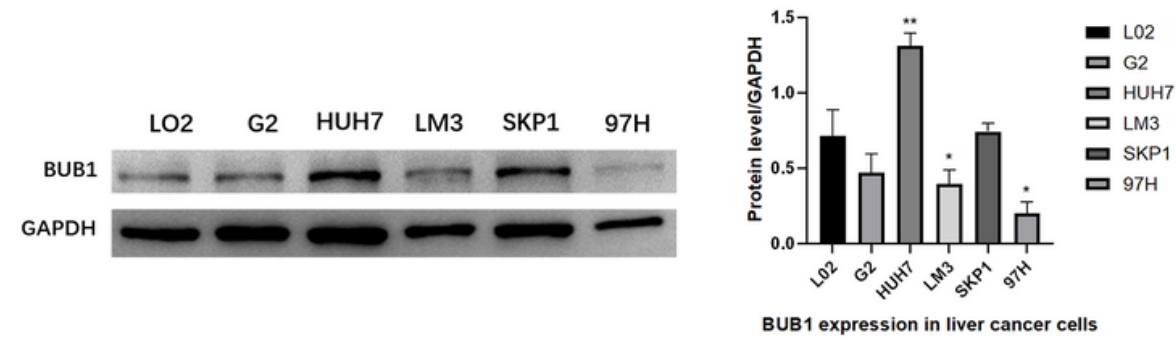

Figure 9

Expression of BUB1 in LIHC. (A) IHC indicated that BUB1 expression was higher in cancer tissues than that in normal liver tissues (original magnification 100x). (B) Kaplan-Meier analysis revealed that LIHC 
patients with high BUB1 expression had poor DFS $(p<0.05)$. (C) WB revealed that BUB1 expression was higher in $\mathrm{HuH7}$ and SKP1 cells than that in other LIHC cell lines.

A

HUH-7

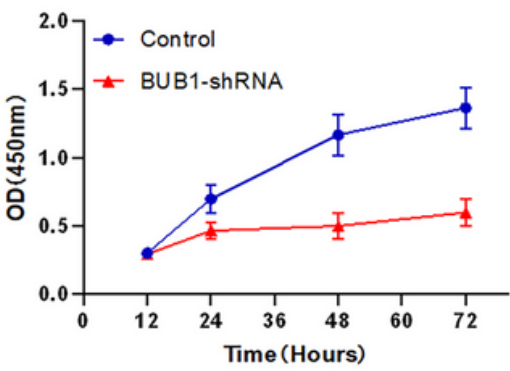

B

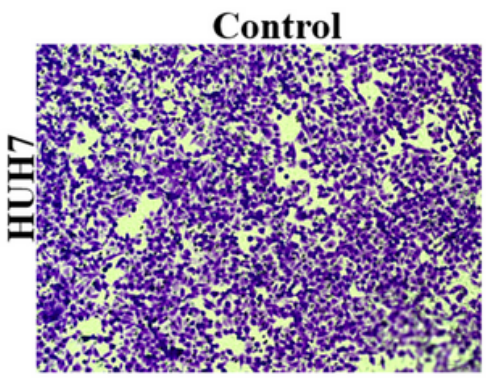

Control

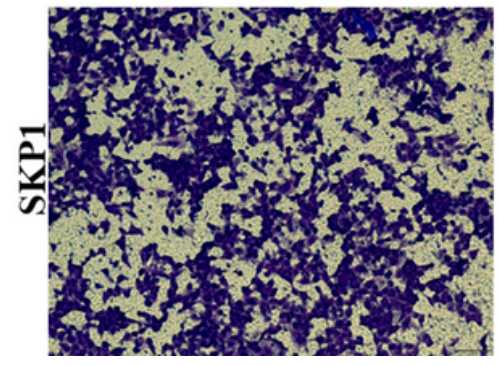

SKP-1

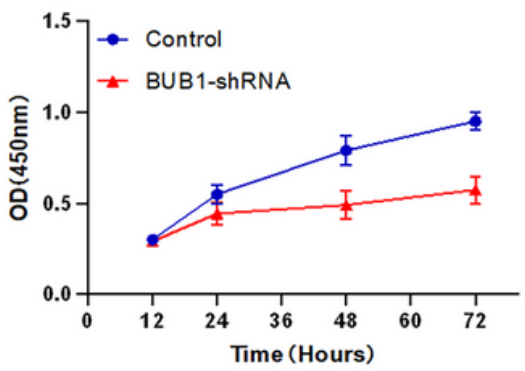

BUB1-shRNA
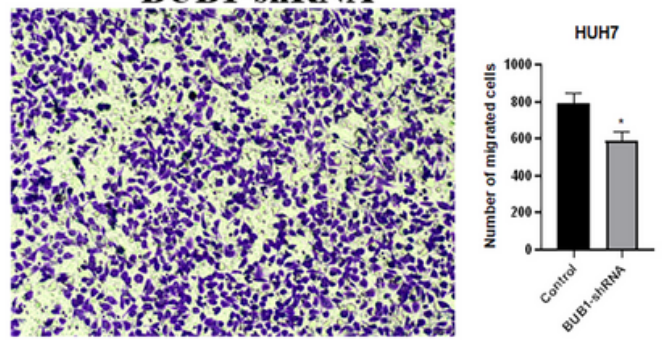

BUB1-shRNA

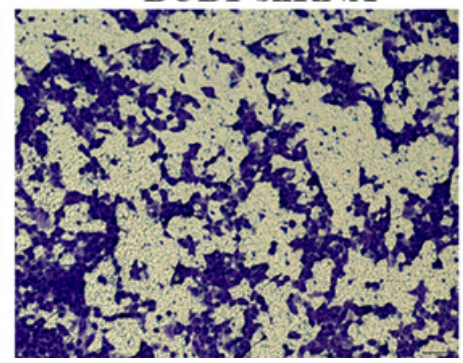

SKP1

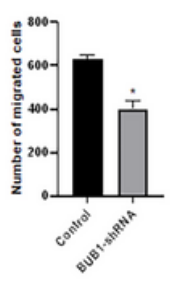

C
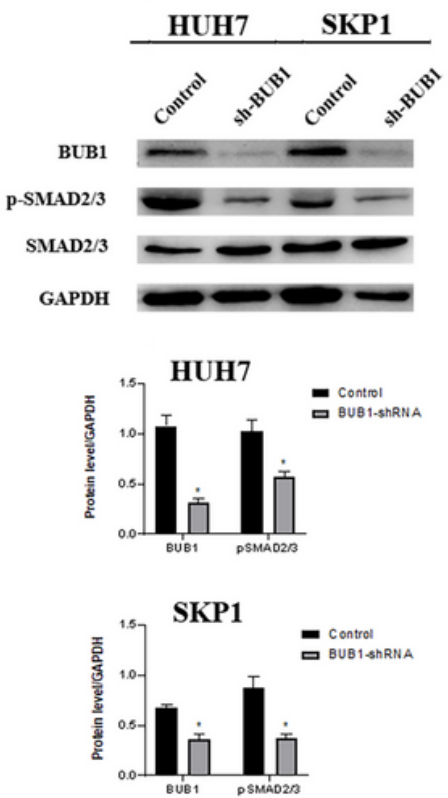

Figure 10

The role of BUB1 in HuH7 and SKP1 cells. (A) CCK-8 assay revealed a reduced proliferation ability of cells with BUB1 knockdown compared with that in control cells at $24 \mathrm{~h}, 48 \mathrm{~h}$, and $72 \mathrm{~h}$. (B) Transwell migration assays revealed a reduced vertical migration ability of cells with BUB1 knockdown compared with that in control group. (C) WB revealed that BUB1 knockdown significantly downregulated the expression of $p$ SMAD2 and p-SMAD3. 
A
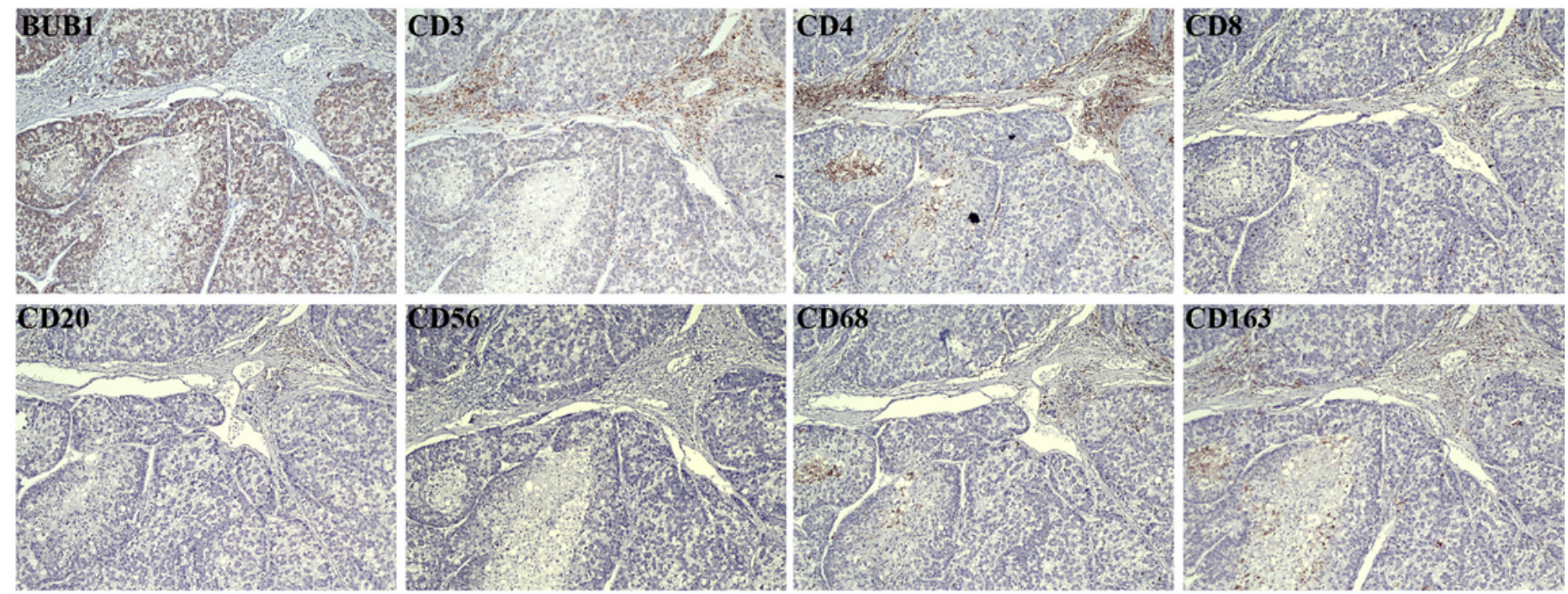

B
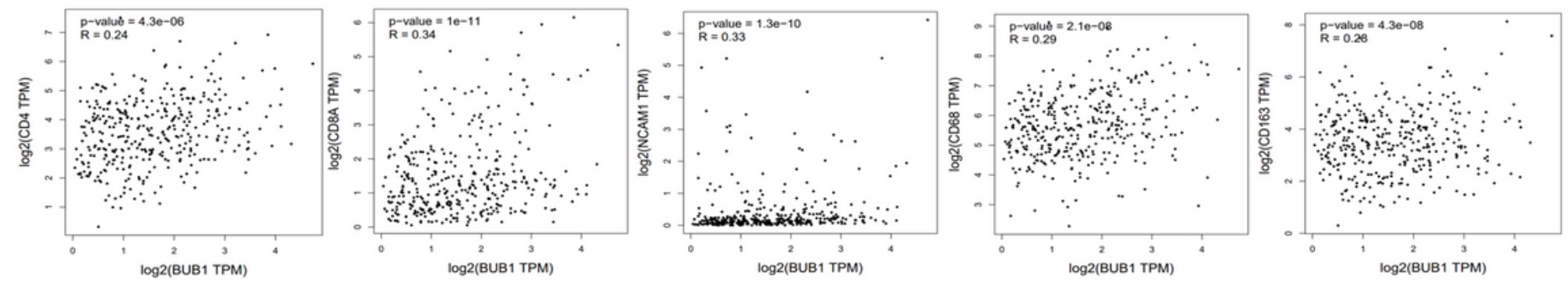

Figure 11

The role of BUB1 in LIHC immune infiltration. (A) IHC of BUB1, CD3, CD4, CD8, CD20, CD 56, CD 68, and CD163 in serial section of LIHC tissues (original magnification $\times 100)$. (B) BUB1 expression was correlated with that of CD4, CD8, CD56 (NCAM1), CD68, and CD163 in LIHC tissues $(p<0.05)$. 